\title{
Higher diversity in fungal species discriminates children with type I diabetes mellitus from healthy control
}

This article was published in the following Dove Press journal:

Patient Preference and Adherence

2I April 2016

Number of times this article has been viewed

\author{
Beata Kowalewska' \\ Katarzyna Zorena ${ }^{2}$ \\ Małgorzata Szmigiero- \\ Kawko ${ }^{3}$ \\ Piotr Wąz $\dot{z}^{4}$ \\ Małgorzata Myśliwiec ${ }^{3}$ \\ 'Department of Tropical Medicine and \\ Epidemiology, Institute of Maritime \\ and Tropical Medicine, ${ }^{2}$ Department \\ of Immunology and Environmental \\ Microbiology, ${ }^{3}$ Clinic of Paediatrics, \\ Diabetology and Endocrinology, \\ ${ }^{4}$ Department of Nuclear Medicine, \\ Medical University of Gdańsk, \\ Gdańsk, Poland
}

Objective: To conduct qualitative and quantitative assessment of yeast-like fungi in the feces of children and adolescents with type 1 diabetes mellitus (T1DM) with respect to their metabolic control and duration of the disease.

Materials and methods: The studied materials included samples of fresh feces collected from 53 children and adolescents with T1DM. Control group included 30 age- and sex-matched healthy individuals. Medical history was taken and physical examination was conducted in the two study arms. Prevalence of the yeast-like fungi in the feces was determined as well as their amounts, species diversity, drug susceptibility, and enzymatic activity.

Results: The yeast-like fungi were found in the samples of feces from $75.4 \%$ of T1DM patients and $70 \%$ controls. In the group of T1DM patients, no correlation was found between age (Rs=0.253, $P=0.068$ ), duration of diabetes ( $\mathrm{Rs}=-0.038, P=0.787)$, or body mass index (Rs $=0.150, P=0.432$ ) and the amount of the yeast-like fungi isolated in the feces. Moreover, no correlation was seen between the amount of the yeast-like fungi and glycated hemoglobin (Rs=0.0324, $P=0.823$ ), systolic blood pressure ( $\mathrm{Rs}=0.102, P=0.483)$, or diastolic blood pressure $(\mathrm{Rs}=0.271, P=0.345)$.

Conclusion: Our research has shown that children and adolescents with T1DM show higher species diversity of the yeast-like fungi, with Candida albicans being significantly less prevalent versus control subjects. Moreover, fungal species in patients with T1DM turn out to be more resistant to antifungal treatment.

Keywords: children, diabetes mellitus type 1, yeast-like fungi, drug susceptibility, metabolic control, duration of the disease

\section{Introduction}

The overall balance in the composition of gut microbiota has a great impact on the proper functioning of the human immune system. ${ }^{1-3}$ According to the most recent research, the composition of the gut microflora can be an important factor that contributes to the development of autoimmune diseases, including diabetes. ${ }^{1,3}$ Some researchers suggest that the composition of microbiota can considerably differ between diabetic patients and healthy subjects. ${ }^{4-6}$ Moreover, the most recent research suggests that the differences in composition of the bacterial community in diabetic patients may influence the growth of the yeast-like fungi in the human alimentary tract. ${ }^{1}$ There have been many reports published on the composition of the bacterial microflora in diabetic patients whereas studies investigating the occurrence of fungi in the gastrointestinal tract of diabetic patients are scarce. Especially, there are very few data on this issue in children and adolescents with type 1 diabetes mellitus (T1DM). The human
Correspondence: Katarzyna Zorena Department of Immunology and Environmental Microbiology, Medical University of Gdańsk, Dębinki 7, 80-2II Gdańsk, Poland

Tel +4858349 1766

Fax +4858349I766

Email kzorena@gumed.edu.pl
Patient Preference and Adherence 2016:10 591-599

Dovepress

http://dx.doi.org/10.2147/PPA.S97852 (c) (1) (5) ( 2016 Kowalewska et al. This work is published and licensed by Dove Medical Press Limited. The full terms of this license are available at https://www.dovepress.com/terms.php cC. hereby accept the Terms. Non-commercial uses of the work are permitted without any further permission from Dove Medical Press Limited, provided the work is properly attributed. For permission for commercial use of this work, please see paragraphs 4.2 and 5 of our Terms (https://www.dovepress.com/terms.php). 
gastrointestinal tract is a site where the yeast-like fungi live in small amounts as saprofites and may require only a small trigger to exert their harmful effects. ${ }^{7-10}$ However, in a healthy human body with properly maintained immune functions, fungal infections are not likely to develop. Nevertheless, when a factor occurs that results in increasing the number of fungal microorganisms and initiates their penetration deep into the body tissues, symptomatic candidiasis may develop. The fungal colonization itself of the digestive tract does not mean fungal infection. Factors contributing to the development of candidiasis include the amount of yeasts attacking the mucous membrane and fungal virulence, which is dependent on the fungal potential to proliferate, adhere, and produce enzymes and toxic metabolites. ${ }^{11-13}$ Symptomatic candidiasis most often develops in endogenous pathways as a result of a variety of factors. Fungal colonization may be a consequence of factors predisposing to fungal proliferation and growth, may precede the disease, or may precede local symptomatic infection. ${ }^{14}$ Furthermore, the most recent studies have shown that increased quantity of the yeast-like fungi found in the digestive tract of diabetic versus nondiabetic patients is not likely to be associated with the pathogenesis of diabetes but is rather its consequence. ${ }^{3,15}$ The increased amount of fungal microorganisms may not only favor the development of symptomatic fungal disease but may also adversely affect the course of diabetes. As in the available literature only a few reports have been identified on the fungal colonization of the gastrointestinal tract in children, especially diabetic children, the aim of this research was to conduct qualitative and quantitative evaluation of the yeast-like fungi found in the feces of children and adolescents with T1DM with respect to their metabolic control and duration of diabetes.

\section{Subjects and methods}

The study involved 53 adolescent patients (19 girls and 34 boys) with T1DM. All patients were under intensive insulin therapy $(0.9 \pm 0.2 \mathrm{IU}$ of insulin per day $/ \mathrm{kg}$ of body weight). Diabetes was diagnosed according to the Polish Diabetes Association guidelines, which correspond with the guidelines of the World Health Organization. ${ }^{16-17}$ Glycated hemoglobin (HbA1c) was measured with an immunoturbidometric method using a Unimate 3 set (Hoffmann-La Roche AG, Basel, Switzerland). Blood pressure was measured using a 24-hour ambulatory blood pressure monitoring (ABPM) method. ${ }^{18}$ Various sizes of the cuff were used according to age, weight, and arm circumference of the studied subjects. All the ABPM results which had less than $80 \%$ of technically correct measurements were excluded from the study. Threshold values defining the range of normal blood pressure values, prehypertension, and hypertension state were according to the centile tables which took into consideration sex, age, and height centile. Arterial hypertension was diagnosed when mean ABPM values were above the 95th centile for the corresponding age, sex, and height on at least three separate measurements. ${ }^{18}$

The control group consisted of 30 healthy children and adolescents, age- and body mass index (BMI)-matched (16 girls and 14 boys). Patients with T1DM and their matched controls were examined by a pediatrician on the day of collection of the fecal samples. Medical history was taken and physical examination was performed and did not reveal any gastrointestinal complaints in either study group. Moreover, the study participants had not been receiving antibiotics for up to 3 months prior their participation in the study. Children with symptoms of infection or systemic somatic illness other than diabetes mellitus were excluded from the study. Written informed consent was obtained from all children and adolescents participating in the study, or from their parent or guardian. The study was approved by The Ethics Committee of The Medical University of Gdańsk (no NKBBN/125/2014) and the investigation was carried out in accordance with the principles of the Declaration of Helsinki as revised in 1996.

\section{Yeast-like fungal cultures}

The study materials were samples of fresh feces collected from the study participants. The feces were collected in sterile containers and provided to the laboratory on the day of collection. On the same day, the samples were used to establish cultures. In order to isolate and enumerate yeastlike fungal colonies in $1 \mathrm{~g}$ feces, quantitative cultures on Sabouraud Dextrose Agar were used. Fecal suspensions in normal saline in serial dilutions 1:10, 1:100, 1:1,000, and $1: 10,000$ were prepared. Five growth lines were drawn on the Sabouraud medium plate. On the first line, $10 \mu \mathrm{g}$ feces was equally spread. Then, $10 \mu \mathrm{g}$ of each fecal dilution was plated and equally spread on each subsequent line, respectively. The cultures were incubated for 72 hours at $37^{\circ} \mathrm{C}$. Then, the number of fungal colonies that had grown on the plate were counted. According to the plate dilution, the number was converted into colony forming units in $1 \mathrm{~g}$ feces. Growth units were established as $10^{3}, 10^{4}, 10^{5}$, and $10^{6} \mathrm{CFU}$.

In order to identify whether the tested samples contained more than one Candida species, the feces was additionally plated on the CHROMagar medium (Graso, Starogard Gdański, Poland). The cultivated fungi were identified according to their morphology and biochemical properties, and tested with commercial API $20 \mathrm{C}$ AUX 
assays (BioMerieux, Paris, France) as instructed by the manufacturer.

\section{Yeast-like fungal drug susceptibility testing} Drug susceptibility of the isolated strains was tested with FUNGITEST ${ }^{\circledR}$ kit (BIO-RAD, Marnes-la-Coquette, France) designed to test the growth of yeast-like fungi in the presence of six drugs at two concentration levels each 5-fluorocitosine ( 2 and $32 \mu \mathrm{g} / \mathrm{mL}$ ), amphotericin B (2 and $8 \mu \mathrm{g} / \mathrm{mL})$, miconazole $(0.5$ and $8 \mu \mathrm{g} / \mathrm{mL})$, ketoconazole $(0.5$ and $4 \mu \mathrm{g} / \mathrm{mL})$, itraconazole $(0.5$ and $4 \mu \mathrm{g} / \mathrm{mL})$, and fluconazole ( 8 and $64 \mu \mathrm{g} / \mathrm{mL}$ ). The test was conducted according to the manufacturer's instructions.

\section{Enzymatic activity testing}

Enzymatic activity of the isolated strains was investigated with the API ZYM system (BioMerieux) according to the manufacturer's instructions. The API ZYM test contains substrates to detect activities of 19 hydrolases.

\section{Statistical analyses}

Statistical analyses were performed with the RKWard Data Analysis Tool Version 0.6.1 using the KDE Development Platform 4.13.3. Quantitative variables were expressed as arithmetic mean and standard deviations. Qualitative variables were presented as numbers and percentages. The Shapiro-Wilk $W$ test was used to verify whether the quantitative variable came from normally distributed population. To investigate differences for the two data sets, Snedecor's $F$-test for homogeneity of variances was performed. When inhomogeneity was found, Wilcoxon-Mann-Whitney $U$-test was used to confirm equality of mean. In case of homogeneity, Student's $t$-test was used for two means. For larger data set, the analysis of variance was conducted with Bartlett's test (more advanced form of Snedecor's $F$-test for a bigger number of samples). When inhomogeneity of variance was detected, differences between the study groups were tested with Kruskal-Wallis test. For groups with homogeneous variance, analysis of variance test was applied. Moreover, chi-square test for independence was used for qualitative variables. To assess the strength and direction of the relationship between two variables, a correlation analysis was performed with the calculation of Pearson's and Spearman's correlation coefficients. Probability value $P$, that is, the level of significance, was established as 0.05 .

\section{Results \\ Clinical characteristics of the study participants}

Clinical characteristics of the studied patients with T1DM as well as the control healthy subjects are presented in Table 1. The study involved 53 children and adolescents with T1DM aged 10.9 \pm 3.9 years, and 30 healthy children and adolescents, age range $10.3 \pm 4.9$ years. The group of patients with T1DM showed significantly higher HbA1c levels versus control subjects, $P=0.0000$. No significant differences were seen in age $(P=0.588)$, sex $(P=0.187)$, BMI $(P=0.635)$, or systolic and diastolic blood pressure in T1DM children versus healthy control subjects.

No yeast-like fungi were grown in fecal samples of 13 T1DM children and nine control subjects. The culture was considered negative. In fecal samples of 40 T1DM patients and 21 control subjects, yeast-like fungal growth was detected in the amount of $10^{3}-10^{6} \mathrm{CFU}$. However, the differences were not statistically significant $(P=0.777)$ (Table 2$)$.

\section{Qualitative evaluation of yeast species in fecal samples of TIDM children and control subjects}

Out of T1DM patients whose 40 fecal samples were yeast-like fungus positive, 33 (82.5\%) cultures contained one species of the yeast-like fungi and in seven T1DM (17.5\%) patients,

Table I Clinical characteristics of patients with TIDM and healthy control subjects

\begin{tabular}{llll}
\hline Parameters & Patients with TIDM & Healthy control subjects & Statistical significance \\
\hline Age (years) & $10.9 \pm 3.9$ & $10.3 \pm 4.9$ & $P=0.588$ \\
Sex (girls/boys) $(\mathrm{n})$ & $19 / 34$ & $16 / 14$ & $P=0.187$ \\
HbAlc $(\%)$ & $7.69 \pm 1.23$ & $4.32 \pm 0.87$ & $P=0.0000 *$ \\
Duration of diabetes (years) & $4.96 \pm 3.03$ & - & - \\
BMl $\left(\mathrm{kg} / \mathrm{m}^{2}\right)$ & $21.4 \pm 2.2$ & $20.4 \pm 1.6$ & $P=0.635$ \\
SBP $(\mathrm{mmHg})$ & $116.0 \pm 10.0$ & $112.0 \pm 9.0$ & $P=0.465$ \\
DBP $(\mathrm{mmHg})$ & $70.0 \pm 10.0$ & $68 \pm 8.0$ & $P=0.571$ \\
\hline
\end{tabular}

Notes: *Patients with TIDM versus healthy control subjects. The results are presented as mean \pm SD, significance $(P<0.05)$.

Abbreviations: TIDM, type I diabetes mellitus; HbAlc, glycated hemoglobin; BMI, body mass index; SBP, systolic blood pressure; DBP, diastolic blood pressure; $\mathrm{SD}$, standard deviation. 
Table 2 Quantitative evaluation of yeast-like fungal colony growth in fecal samples of TIDM children and control subjects

\begin{tabular}{llll}
\hline $\begin{array}{l}\text { Yeast-like } \\
\text { fungi }\end{array}$ & $\begin{array}{l}\text { TIDM children } \\
\mathbf{n}=\mathbf{5 3}\end{array}$ & $\begin{array}{l}\text { Control } \\
\text { subjects } \mathbf{n}=\mathbf{3 0}\end{array}$ & $\begin{array}{l}\text { Statistical } \\
\text { significance }\end{array}$ \\
\hline Negative & 13 & 9 & $P=0.777$ \\
Positive & 40 & 21 & \\
\hline
\end{tabular}

Abbreviation: TIDM, type I diabetes mellitus.

two yeast-like fungal species were grown in one culture. In the control group of 30 nondiabetic children, fungus-positive cultures were seen in $21(70 \%)$ children. The differences were not statistically significant $(P=0.106)$ (Table 3$)$. In all of the fungus-positive samples, single-species cultures were obtained. In total, 47 strains of the yeast-like fungi were grown in the fungus-positive fecal samples of T1DM children (Figure 1A). The isolated strains belonged to nine species. Candida spp. accounted for $83 \%$ of the isolated strains and the remaining 17\% were strains of the other yeast-like fungi. Among the isolated strains, Candida albicans was the main species and made up $62 \%$ of all of the grown strains. The other Candida species that were grown included C. krusei-9\% of all of the isolated strains; C. famata $-6 \%$; and C. parapsilosis, C. lusitaniae, and C. guilermondii $-2 \%$ of all of the isolated strains each. The remaining yeast-like fungal species isolated were Rhodotorula spp. (9\%), Geotrichum spp. $(6 \%)$, and Saccharomyces spp. (2\%). In the materials from control group, 21 yeast-like fungal strains were detected (Figure 1B). The isolated strains belonged to four species. Among the isolated strains, the main species was $C$. albicans which accounted for $85 \%$ of all of the yeast-like fungal strains grown. Moreover, C.famata and C. tropicalis were detected in this group accounting for $5 \%$ of all strains. The last fungal species detected was Rhodotorula spp., not being a member of the Candida genus, which accounted for $5 \%$ of isolates (Figure 1B).

\section{Correlation between the amount of the yeast-like fungi and clinical parameters in TIDM children}

The aim of the next part of the study was to investigate the relationship between the amount of the yeast-like fungi and

Table 3 Frequency of single and multiple species in cultures from the stool samples in children with TIDM and control subjects

\begin{tabular}{llll}
\hline Yeast-like fungi & $\begin{array}{l}\text { TIDM } \\
\text { children }\end{array}$ & $\begin{array}{l}\text { Control } \\
\text { subjects }\end{array}$ & $\begin{array}{l}\text { Statistical } \\
\text { significance }\end{array}$ \\
\hline Single species in culture & 33 & 21 & $P=0.106$ \\
Two species in culture & 7 & 0 & \\
\hline
\end{tabular}

Abbreviation: TIDM, type I diabetes mellitus. clinical parameters in T1DM children versus control group. Therefore, Spearman's rank correlation coefficient was calculated and the results are presented in Figure 2.

In the group of T1DM children, no statistically significant correlations were seen between age $(\mathrm{Rs}=0.253$, $P=0.0681$ ), duration of diabetes ( $\mathrm{Rs}=-0.0388, P=0.787$ ), or BMI (Rs=0.150, $P=0.432$ ) and of the yeast-like fungi cultivated from the feces. Moreover, no correlations were seen between the yeast-like fungi and level of $\mathrm{HbA} 1 \mathrm{c}(\mathrm{Rs}=0.0324$, $P=0.823$ ), systolic blood pressure ( $\mathrm{Rs}=0.102, P=0.483$ ), or diastolic blood pressure $(\mathrm{Rs}=0.271, P=0.345)$ (Figure 2).

\section{Quantitative evaluation of the yeast-like fungi in TIDM children according to their metabolic control and duration of diabetes}

The next step involved an attempt to identify whether the level of metabolic control and duration of diabetes may affect the dynamics of the yeast-like fungi - at the range of $10^{3} \mathrm{CFU} / \mathrm{g}$ to $10^{6} \mathrm{CFU} / \mathrm{g}$ in the tested fecal samples from T1DM children. Duration of diabetes was stratified into $<5$ and $>5$ years. Metabolic control was assessed as $\mathrm{HbA} 1 \mathrm{c}<7.5 \%$ and $>7.5 \%$.

In fecal samples collected from T1DM children, no correlation was detected between the yeast-like fungi in the range of $10^{3} \mathrm{CFU} / \mathrm{g}$ to $10^{6} \mathrm{CFU} / \mathrm{g}$ and $\mathrm{HbA} 1 \mathrm{c}$ levels $<7.5 \%$ and $>7.5 \%$.

In fecal samples collected from T1DM children, no correlation was detected between the yeast-like fungi in the range of $10^{3} \mathrm{CFU} / \mathrm{g}$ to $10^{6} \mathrm{CFU} / \mathrm{g}$ and duration of diabetes $<5$ and $>5$ years.

\section{Investigation of drug susceptibility of C. albicans isolates from fecal samples in TIDM patients}

Susceptibility of the isolated strains of $C$. albicans to the following six antifungal drugs was tested: 5-fluorocytosine, amphotericin, miconazole, ketoconazole, itraconazole, and fluconazole. In the groups of T1DM children and control subjects, $100 \%$ of C. albicans strains were susceptible to 5-fluorocytozine and amphotericin. Only 28\% of $C$. albicans strains were susceptible to itraconazole in T1DM patients and 54.5\% in healthy control subjects (Figure 3A and B).

Candida non-albicans strains isolated from the tested samples of T1DM children appeared to be less susceptible to the tested drugs than C. albicans strains: only $11 \%$ were susceptible to itraconazole, $33 \%$ to miconazole, 

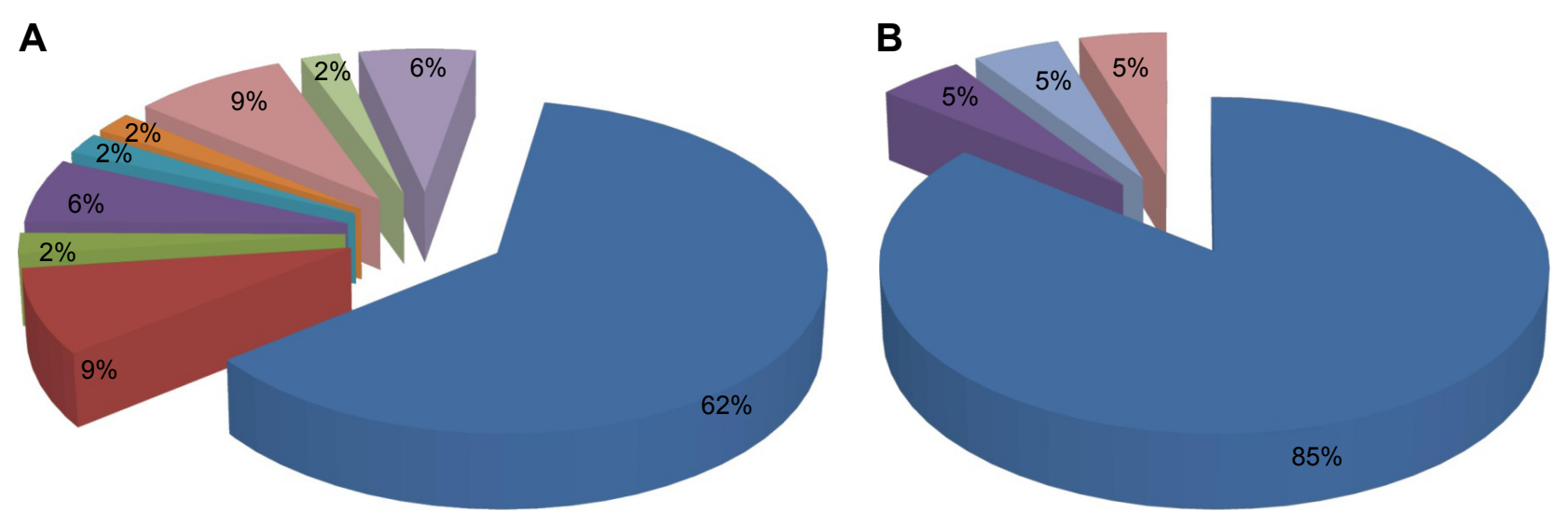

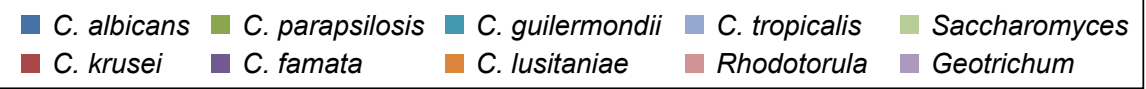

Figure I The amount of the yeast-like fungal species in type I diabetes mellitus children $(\mathbf{A})$ and the control subjects (B).

$56 \%$ to fluconazole, $67 \%$ to 5 -fluorocytosine, and $89 \%$ to amphotericin and ketoconazole (Figure 4).

In the samples collected form T1DM children, $28 \%$ of C. albicans strains were susceptible to all of the tested drugs, $61 \%$ of the strains were intermediate or resistant to one drug, $5.5 \%$ of the strains were susceptible to three of the tested drugs with intermediate sensitivity or resistance to the remaining three drugs, and $5.5 \%$ of the strains were susceptible to as few as two of the tested drugs (Figure 5A). Out of the C. albicans strains isolated from healthy children, $59 \%$ were susceptible to all of the tested drugs, $35 \%$ were susceptible to five drugs, and $6 \%$ were not susceptible to two of the six tested drugs (Figure 5B).

\section{Investigation of enzymatic activity of C. albicans isolates in TIDM children and healthy control subjects}

Tests in the group of T1DM children have shown activities in 14 out of 19 hydrolytic enzymes of $C$. albicans strains isolated from fecal samples. In individual C. albicans strains, activities of nine to 12 enzymes were detected. No activity of lipase, $\alpha$-chymotrypsin, $\beta$-glucosidase, $\beta$-glucuronidase, and $\alpha$-fucosidase was detected. In the group of T1DM children, $C$. albicans strains showed the highest mean activities (mean score $>4$ on the scale of $0-5$ ) of acid phosphatase (mean score of 4.3) and leucine arylamidase (4.8) (25-35 nmol of hydrolyzed substrate). High mean hydrolytic activity ( $>2$ on the scale of $0-5$ ) (10-20 nmol) was seen for $\mathrm{C} 4$ esterase and esterase lipase, valine arylamidase, and $\mathrm{N}$-acetyl-beta-glucosaminidase. C. albicans strains isolated from fecal samples of healthy control subjects showed activities of ten out of 19 tested hydrolases. In individual strains of C. albicans, activities of eight to ten enzymes were detected. No enzymatic activity of C. albicans strains was detected for lipase, $\alpha$-chymotrypsin, trypsin, $\alpha$-galactosidase, $\beta$-galactosidase, $\beta$-glucuronidase, $\beta$-glucosidase, $\alpha$-fucosidase, and $\alpha$-mannosidase. The

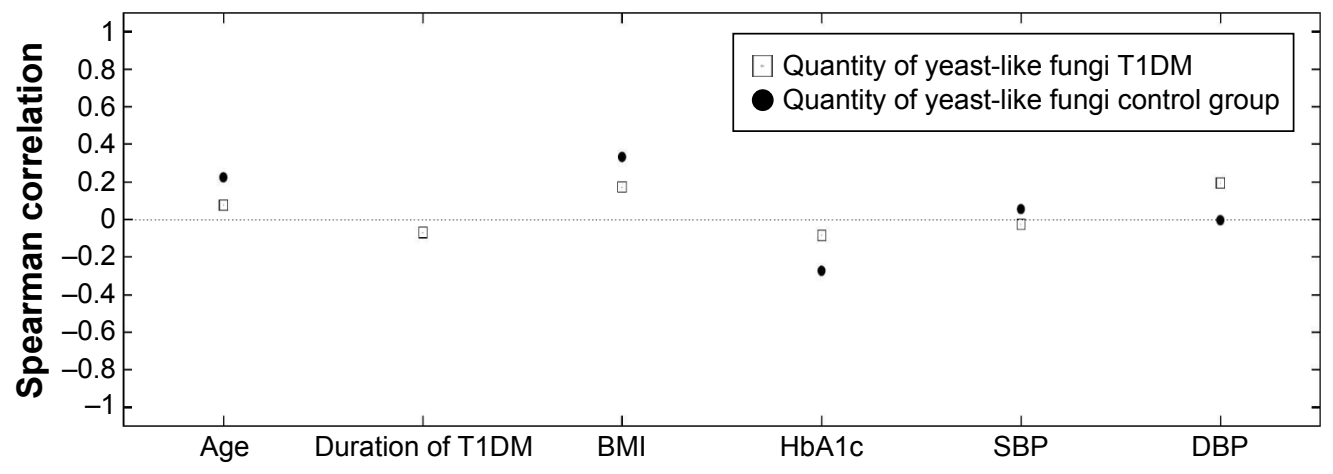

Figure 2 Relationship between the amount of the yeast-like fungi and clinical parameters in the study groups.

Abbreviations: TIDM, type I diabetes mellitus; BMI, body mass index; HbAIc, glycated hemoglobin; SBP, systolic blood pressure; DBP, diastolic blood pressure. 

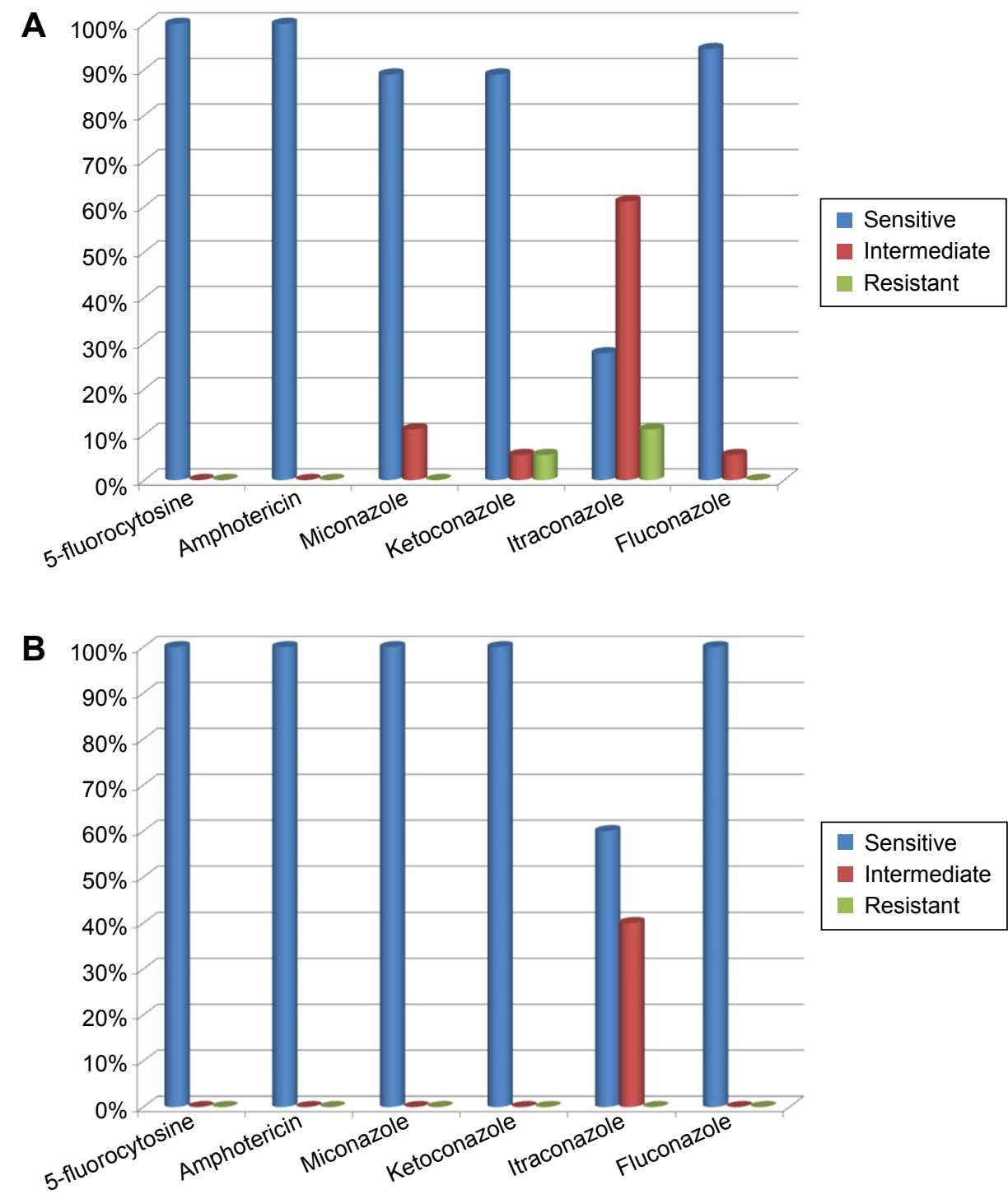

Figure 3 Drug susceptibility of Candida albicans strains isolated from fecal samples of type I diabetes mellitus children (A) and control subjects (B).

highest activity (mean score $>3$ on the scale of $0-5$ ) was detected for acid phosphatase (4.2) and leucine arylamidase (4.4), followed by $\mathrm{N}$-acetyl- $\beta$-glucosaminidase (3.1), esterase (2.1), and esterase lipase (2.2) and $\alpha$-glucosidase (2.2).

\section{Discussion}

In our study, the yeast-like fungal growth in fecal samples was detected in 40 (75.4\%) T1DM patients and 21 (70\%) control subjects. Surprisingly, our analyses have shown high prevalence of yeast colonies occurring both in type 1 diabetic patients and healthy children. Some authors of the previous studies reported yeast-like fungal colonization in $91 \%$ of T1DM children ${ }^{19}$ whereas in other reports the percentage of yeast-like fungus positive diabetic patients was lower and approximated $40 \% .{ }^{1,20}$ Consistent with our results, also in children with malignant disease no significant differences in yeast-like fungal isolates were detected versus healthy children. ${ }^{21}$ The authors suggested that fungal colonization may depend on the patient's individual predisposing factors. ${ }^{21}$

In our studies, in $17.5 \%$ of T1DM patients, two species of yeast-like fungi were detected whereas in healthy children only one species occurred. Moreover, although in the studied sample of T1DM children the dominant species was C. albicans, which accounted for $62 \%$ of all of the yeastlike fungal isolates, its prevalence was lower versus healthy children. The results of our study indicate increasing prevalence of C.non-albicans species. The decreasing prevalence of C. albicans in favor of other Candida species has already been reported by other authors in their most recent works..$^{19,22-25}$

The next step of our research was to investigate the relationship between the amount of the yeast-like fungi and clinical parameters in T1DM children and control subjects. 


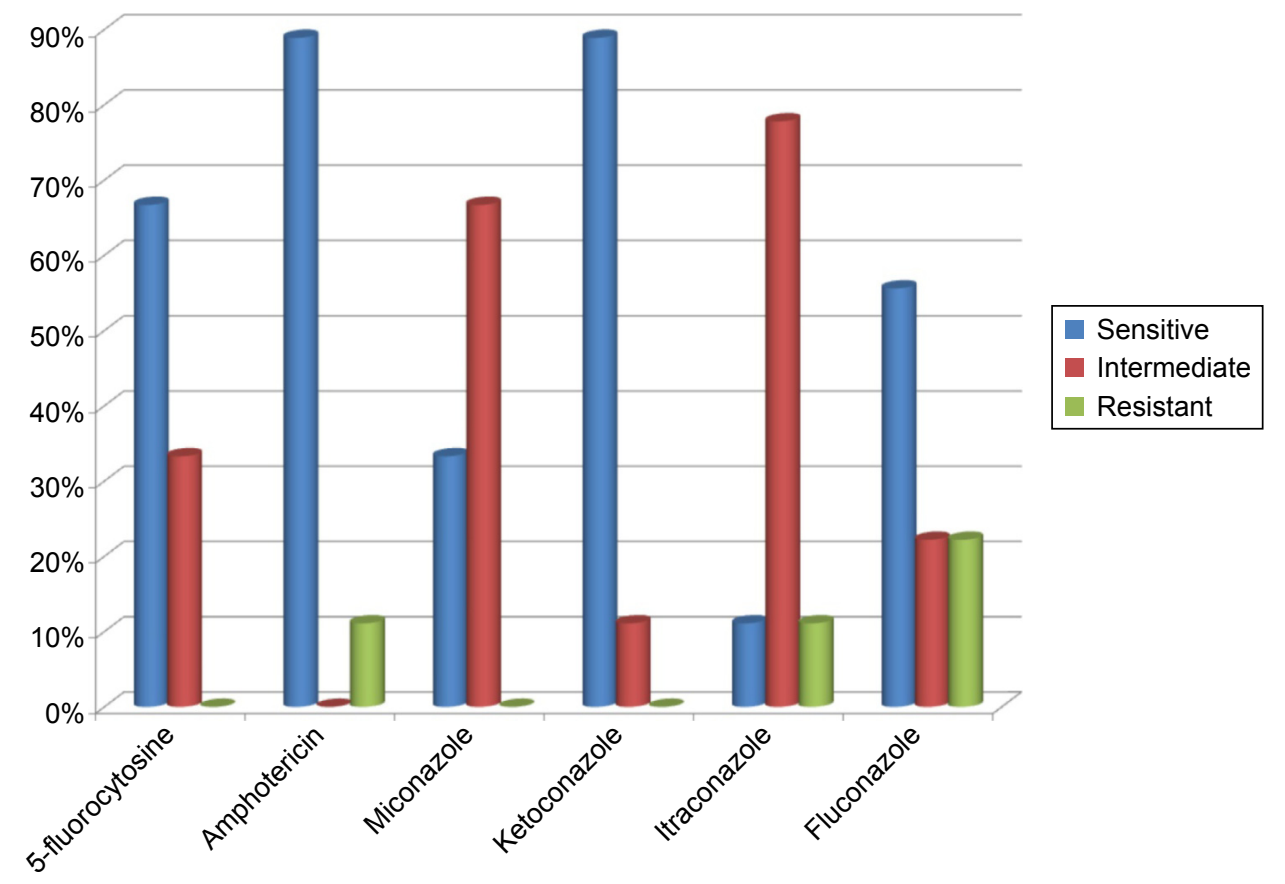

Figure 4 Drug susceptibility of Candida spp. strains in patients with type I diabetes mellitus.

In the group of T1DM children, no correlation was seen between the patient's age, duration of diabetes, or BMI and the amount of the yeast-like fungi in the fecal samples. Moreover, no correlation was detected between the amount of the yeast-like fungi and systolic and diastolic blood pressure and the level of metabolic control. Consistent with our results, other authors in their recent report described no correlation between the amount of the yeast-like fungi and level of HbAlc. ${ }^{15}$ However, the prevalence of Candida spp. was studied in fecal samples from adult patients with T1DM and type 2 diabetes mellitus (T2DM). Therefore, no correlation was detected between the level of fungal colonization and level of metabolic control either in T1DM children or T1DM and type 2 diabetes mellitus adults. ${ }^{15} \mathrm{We}$ believe that in patients with a short history of diabetes of $<5$ years and good metabolic control, the prevalence of the yeast-like fungi in the digestive tract is not significantly increased. In our study, the amount of the yeast-like fungi was not increased
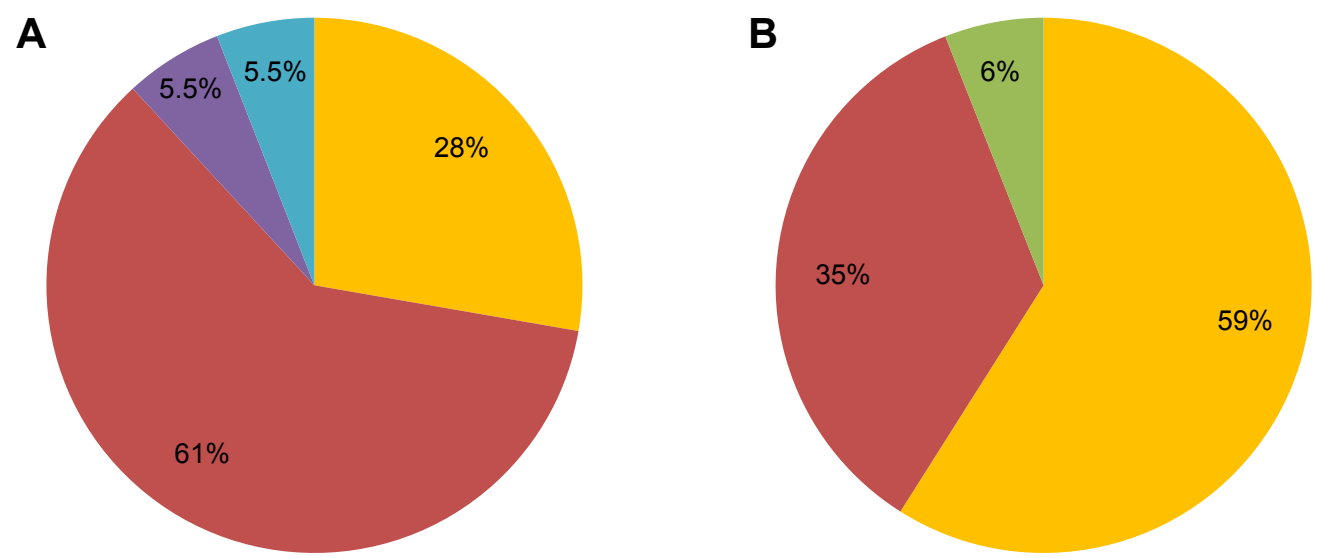

$\begin{aligned} & \text { Susceptible to all of the tested drugs } \\ & \text { Intermediate or resistant to one drug }\end{aligned}$ Intermediate or resistant to two of the tested drugs
Intermediate or resistant to more than three drugs

Figure 5 Comparison of Candida albicans strains susceptible, intermediate, and resistant to the six tested antifungal drugs in children with type I diabetes mellitus (A) and healthy control subjects (B). 
in T1DM children versus healthy control subjects. This could be related to the fact that the disease in T1DM children was of short duration and with good metabolic control but also to the fact that children had not complained of any gastrointestinal problems and had not been treated with any antibiotics for up to 3 months prior to their fecal sample collection. However, it seems interesting that in the samples collected from T1DM children, a higher diversity of fungal species was observed versus control subjects. In the studied group of T1DM children versus nondiabetic children, there were more C. albicans strains with lower susceptibility to the tested antifungal drugs. What is of concern is the relatively high number of strains showing low susceptibility or resistance to itraconazole and lower number of strains susceptible to fluconazole and ketoconazole in T1DM children. In the very few studies that are currently available in T1DM children, no increase in the number of $C$. albicans strains with lower susceptibility to the studied drugs was reported. Only 4\% of $C$. albicans strains showed reduced susceptibility to fluconazole and $6 \%$ were resistant to ketoconazole. ${ }^{26}$ However, increased prevalence of drug-resistant Candida strains was observed in children with malignant diseases.

In a study, Gualco et $\mathrm{al}^{27}$ have observed a higher percentage of itraconazole-resistant $C$. albicans obtained in children versus adults. There is evidence in the literature that drug resistance of Candida spp. is increasing. For example, Bremenkamp et $\mathrm{al}^{28}$ showed that $47.2 \%$ of Candida strains isolated from the oral cavity of T1DM patients were resistant to ketoconazole. Our studies have also shown an increased number of Candida spp. strains resistant to the studied drugs. Isolation of increasing numbers of strains with decreased drug susceptibility may significantly impact the conditions in which factors contributing to candidiasis occur and may cause difficulties in treatment. ${ }^{29}$ The more the risk factors, such as impaired immunity or the use of antibiotics, occur in a patient and the higher the pathogenic potential of the isolated strain, the higher the risk of severe candidiasis-related problems.

Enzymatic activity is among the factors that strongly affects fungal virulence. Candida spp. can produce numerous enzymes that determine the nature and course of the infection. Enzymatic activity may contribute to disruption of the balance between the fungus and host. Lipases are considered important in the first phase of infection; proteases can damage epithelial cells and allow the fungi to invade deep into the tissues. ${ }^{14}$ Enzymatic activity testing in the yeast-like fungi obtained from the oral cavity has shown higher protease activities in strains cultured from specimens collected from diabetic patients versus control subjects. ${ }^{30}$ Higher enzymatic activity of strains obtained from T1DM children may contribute to their higher virulence and may confer more risks in conditions of impaired defense mechanisms in a patient.

In conclusion, the yeast-like fungi isolated from fecal samples of T1DM children showed more species diversity versus those isolated from control samples. Moreover, more C. albicans strains in this group showed lower susceptibility to the tested drugs and higher enzymatic activity. Mycological tests, including quantitative evaluation of fecal samples, should be considered as part of a diagnostic approach in T1DM children. When yeast-like fungi are isolated, their drug susceptibility should be determined as there are an increasing number of strains resistant to the commonly used antifungal drugs.

\section{Acknowledgment}

This work was supported by The State Committee for Scientific Research ST-120 (Medical University of Gdańsk).

\section{Disclosure}

The authors report no conflicts of interest in this work.

\section{References}

1. Soyucen E, Gulcan A, Aktuglu-Zeybek AC, Onal H, Kiykim E, Aydin A. Differences in the gut microbiota of healthy children and those with type 1 diabetes. Pediatr Int. 2014;56(3):336-343.

2. Vaarala O, Atkinson MA, Neu J. The "perfect storm" for type 1 diabetes - the complex interplay between intestinal microbiota, gut permeability, and mucosal immunity. Diabetes. 2008;57:2555-2562.

3. Dunne JL, Triplett EW, Gevers D, et al. The intestinal microbiome in type 1 diabetes. Clin Exp Immunol. 2014;177(1):30-37.

4. Boerner BP, Sarvetnick NE. Type 1 diabetes: role of intestinal microbiome in humans and mice. Ann N Y Acad Sci. 2011;1243:103-118.

5. Vaarala O. Gut microbiota and type 1 diabetes. Rev Diabet Stud. 2012;9:251-259.

6. Murri M, Leiva I, Gomez-Zumaquero JM, et al. Gut microbiota in children with type 1 diabetes differs from that in healthy children: a case-control study. BMC Med. 2013;11:46.

7. Bernhardt H, Knoke M. Mycological aspects of gastrointestinal microflora. Scand J Gastroenterol Suppl. 1997;222:102-106.

8. Kumamoto CA. Inflammation and gastrointestinal Candida colonization. Curr Opin Microbiol. 2011;14(4):386-391.

9. White SJ, Rosenbach A, Lephart P, et al. Self-regulation of Candida albicans population size during GI colonization. PLoS Pathog. 2007;3(12):e184.

10. Huffnagle GB, Noverr MC. The emerging world of the fungal microbiome. Trends Microbiol. 2013;21(7):334-341.

11. Szypowska A, Pańkowska E. Selected parameters of immunological system in children and adolescents with poorly controlled type-1 diabetes and fungal infections. Nowa Pediatria. 2004;2:46-50.

12. Schulze J, Sonnenborn U. Yeasts in the gut: From commensals to infectious agents. Dtsch Arztebl Int. 2009;106(51-52):837-842.

13. Kowalewska B, Kawko M, Zorena K, Myśliwiec M. Yeast-like fungi in the gastrointestinal tract in children and adolescents with diabetes type 1. Pediatr Endocrinol Diabetes Metab. 2015;20(4):170-177.

14. Batura-Gabryel H, Brajer B, Kuźnar B, Młynarczyk W. Can hydrolytic activity of Candida strains isolated from COPD patients change with the basic disease progression? Post Derm Alerg. 2003;3:148-155. 
15. Gosiewski T, Salamon D, Szopa M, Sroka A, Malecki MT, Bulanda M. Quantitative evaluation of fungi of the genus Candida in the feces of adult patients with type 1 and 2 diabetes - a pilot study. Gut Pathog. 2014 15;6(1):43

16. Position of the Polish Diabetes Association, "Clinical Recommendations for treatment of patients with diabetes." Clin Diabetol. 2014;3Supplement A3-A5.

17. WHO: Definition. Diagnosis and Classification of Diabetes Mellitus and its Complications. Report of a WHO Consultation. Part 1. Diagnosis and Classification of Diabetes Mellitus. Geneva: WHO; 2006.

18. National High Blood Pressure Education Program Working Group on High Blood Pressure in Children and Adolescents. The fourth report on the diagnosis, evaluation and treatment of high blood pressure in children and adolescent. Pediatrics. 2004;114:555-576.

19. Cisło M, Wąsik-Kuprianowicz A, Baran E, Noczyńska A. Occurrence of yeast and moulds in the digestive tract in children with diabetes type 1. Part II. Qualitative and quantitative fungi evaluation in the stool samples. Med Mycol. 2003;10(3):193-198.

20. Nowakowska M, Jarosz-Chobot P. [Bacterial and fungal flora in some clinical materials in children with diabetes type 1]. Pediatr Endocrinol Diabetes Metab. 2002;8(2):83-88. Polish [with English Abstract].

21. Gammelsrud KW, Sandven P, Høiby EA, Sandvik L, Brandtzaeg P, Gaustad P. Colonization by Candida in children with cancer, children with cystic fibrosis, and healthy controls. Clin Microbiol Infect. 2011;17(12): 1875-1881.

22. Macura A, Witalis J. Fungi isolated from the stool in patients with gastrointestinal disorders in 2005-2009. Przegl Epidemiol. 2010;64: 313-317.
23. Khatib R, Riederer KM, Ramanathan J, Baran J Jr. Faecal fungal flora in healthy volunteers and inpatients. Mycoses. 2001;44(5):151-156.

24. Agirbasli H, Ozcan SA, Gedikoğlu G. Fecal fungal flora of pediatric healthy volunteers and immunosuppressed patients. Mycopathologia. $2005 ; 159(4): 515-520$.

25. Tsai MH, Wang SH, Hsu JF, et al. Clinical and molecular characteristics of bloodstream infections caused by Candida albicans in children from 2003 to 2011. Clin Microbiol Infect. 2015;21(11):1018.e1-e8.

26. Nawrot U, Cisło M, Noczyńska A, et al. Susceptibility to selected antifungal agents of Yeats-like fungi isolated from gastrointestinal tract of diabetes children type 1. Med Mycol. 2006;13(1):35-38.

27. Gualco L, Debbia EA, Bandettini R, et al. Antifungal resistance in Candida spp. isolated in Italy between 2002 and 2005 from children and adults. Int J Antimicrob Agents. 2007;29(2):179-184.

28. Bremenkamp RM, Caris AR, Jorge AO. et al. Prevalence and antifungal resistance profile of Candida spp. oral isolates from patients with type 1 and 2 diabetes mellitus. Arch Oral Biol. 2011;56(6):549-555.

29. Arendrup MC. Candida and candidaemia. Susceptibility and epidemiology. Dan Med J. 2013;60(11):B4698.

30. Rajendran R, Robertson DP, Hodge PJ, Lappin DF, Ramage G. Hydrolytic enzyme production is associated with Candida albicans biofilm formation from patients with type 1 diabetes. Mycopathologia. 2010;170(4):229-235
Patient Preference and Adherence

\section{Publish your work in this journal}

Patient Preference and Adherence is an international, peer-reviewed, open access journal that focuses on the growing importance of patient preference and adherence throughout the therapeutic continuum. Patient satisfaction, acceptability, quality of life, compliance, persistence and their role in developing new therapeutic modalities and compounds to optimize

\section{Dovepress}

clinical outcomes for existing disease states are major areas of interest for the journal. This journal has been accepted for indexing on PubMed Central. The manuscript management system is completely online and includes a very quick and fair peer-review system, which is all easy to use. Visit http://www. dovepress.com/testimonials.php to read real quotes from published authors. 